\title{
Translation of 3D bioprinting into medical practice: learning from related fields to focus our efforts
}

\author{
"...medical technology researchers must take a translational \\ perspective in order that their ideas reach patients. For 3D \\ bioprinting, there is a particular need to tackle regulatory \\ science for manufacturing..."
}

First draft submitted: 21 December 2015; Accepted for publication: 22 December 2015; Published online: 22 January 2016

Our community has a great and important vision - the routine use of customized living 3D bioprinted products that will transform medicine by permitting new approaches to patient care. Conferences, publications and this new journal exemplify both the promise and the scientific energy of the field.

Our hope is that we will be able to build upon earlier successes. 3D printing is becoming routine in surgery planning. This is a consequence of the energy of the people involved, clear application pull and a commercial environment with business led leadership - in particular a strong company that has shaped and pioneered the field. It is also a consequence of the engineering community understanding its fit with the practice of medicine, those who practice medicine and the necessary regulatory pathway. Conventional 3D printed materials are now beginning to be applied in medical device settings of increasing complexity. This is once again a consequence of a clearer understanding of the principles of the regulatory pathway that allows them to be used - that the properties of the materials and structures made by $3 \mathrm{D}$ printing/additive manufacturing are equivalent to the properties achieved for the product via conventional manufacturing. We are also seeing significant benefit from the application of $3 \mathrm{D}$ printing techniques to personalized and customized prosthetics, delivered by a regulatory pathway for manufacturing extending that of traditional craft processes carried out in hospital settings.
We have demonstrated that we understand our fit as a community with medical device regulatory pathways for both products and manufacturing, and we have understood how we add value to the practice of medicine and have identified our fit with the practice of medicine.

We do, however, need to be careful on how excited we get about our more visionary products - they are much more complex and in many cases have 3D printed forms, include living cells and complex micro- and macrostructures, and are customized - ultimately with the ambition of creating organs for transplant that are physiologically equivalent to human organs. Many also see the logical setting for their manufacture as in a number of specialist hospitals rather than in a conventional centralized 'factory'. As a community we need to be prepared for the effort that will be required to translate these new and emerging technologies from the laboratory bench to the bedside - this is the only way we will bring benefit to a large number of patients.

Translation of a medical technology requires some fundamental questions to be addressed by a developer taking account of the perspectives of a range of important stakeholders:

- Technology - does your product work and can you make it?

- Regulation - how is it interpreted and applied to your product, product approval

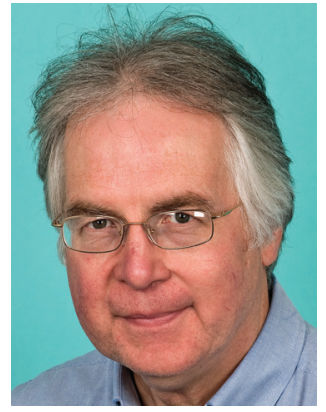

David J Williams

Professor of Healthcare Engineering, Loughborough University, LE11 3TU, UK D.J.Williams2@lboro.ac.uk 
including clinical trials, and Good Manufacturing Practice (GMP) manufacturing?

- Reimbursement and cost of goods - is anybody going to pay for it? This is especially important in our cost-constrained healthcare systems. Will we be able to generate a profitable margin?

- Adoption and clinical pull - will anybody use it and will they be able to persuade somebody to buy it?

- Will anybody invest, and under what business model - pharmaceutical, device, combination product, service or procedure?

For 3D bioprinted products, some of these questions are particularly testing and will particularly exercise a community with manufacturing roots like 3D printing. Significant challenges fundamentally derive from the likely regulatory classifications of 3D bioprinted products if they include living cells within some form of 'scaffold'. In the USA, these are likely to be combination products including a biologic and in the EU, combination products including an Advanced Therapeutic Medicinal Product (ATMP). The regulatory pathways for ATMPs using cells alone are particularly complex and challenging to navigate. In the $3 \mathrm{D}$ printed case, there are the added complexities to the product regulatory pathway of a scaffold including a macro- or microstructure, essentially resulting in the combination product designation; there are also many challenges in customized regulated (GMP) manufacturing.

It is important to recognize that ambiguities and uncertainties in regulation considerably impacted the closely related field of cell therapy, these ambiguities only being resolved in Europe in 2007. Getting regulatory clarity and moving from 'case by case' product regulation is still an important focus for the cell therapy; this was product focused at first but is now increasingly addressing manufacturing. 3D bioprinting requires similar clarity.

Key manufacturing complexities arise from customization, in particular the computer-aided design computer-aided manufacturing (CAD-CAM) model compilation and code generation steps that generate the instructions for the printing machine. The sensible expectation that such products will also be delivered in a number of different and distributed clinical centers is also complex to address when the expectation is that the products will be the same in the different hospitals.

\section{References}

1 Therman-Newall J, Petzing J, Williams D. Quantification of biological variation in blood-based therapy - a summary of a meta-analysis to inform manufacturing in the clinic. Vox Sanguinis 109(4), 394-402 (2015).
These are extremely challenging under GMP (in the $\mathrm{EU}$, and cGMP [c is current] in the USA) and require demonstrable reproducibility of process - executing the same process in the same way 'again and again' - and comparability - ensuring that the process is the same after a change. The key target for reproducibility is the management of biological variation (see ThermanNewall et al. [1]), and for change is the ability to execute the same process in a different site.

However, the most challenging step from a regulated manufacturing perspective is the validation (proving that the process does what it should) of the CADCAM code-generation step to ensure that it consistently delivers 3D printed living product - remember cells are alive and not conventional 'inert' engineering materials with and without customization of geometry. Without this validation we cannot be sure, for example, that we are not creating necrosing (dying) tissue that could be subsequently transplanted. These issues are discussed in full in Hourd et al. [2].

The message of this discussion is that medical technology researchers must take a translational perspective in order that their ideas reach patients. For 3D bioprinting, there is a particular need to tackle regulatory science for manufacturing by addressing research questions raised by the regulatory framework that protects patients. The output of this pattern of work - especially if precompetitive - will inform regulators and industry as they work to develop these products to address clinical need and deliver patient benefit. The scientific community must work together to define the key questions that need to be addressed and reach out to developers, regulators and clinicians to ensure the methods they use to address the questions are endorsed, their results appropriately reviewed and the outcomes fit with evolution in the practice of medicine and relevant clinical pathways - this is the only way research outcomes will be accepted and applied.

Financial \& competing interests disclosure

The author has no relevant affiliations or financial involvement with any organization or entity with a financial interest in or financial conflict with the subject matter or materials discussed in the manuscript. This includes employment, consultancies, honoraria, stock ownership or options, expert testimony, grants or patents received or pending, or royalties.

No writing assistance was utilized in the production of this manuscript.

2 Hourd P, Medcalf N, Segal J, Williams D. A 3D bioprinting exemplar of the consequences of the regulatory requirements on customized processes. Regen. Med. 10(7), 863-883 (2015). 